\title{
Massive Pulmonary Thromboembolism Demonstrated at Necropsy in Japanese Psychiatric Patients Treated With Neuroleptics Including Atypical Antipsychotics
}

\author{
Satoko Hamanaka, MD; Yoshito Kamijo, MD*; Tomonori Nagai, MD**; \\ Katsuyoshi Kurihara, MD**; Katsutoshi Tanaka, $\mathrm{MD}^{\dagger}$; \\ Kazui Soma, MD*; Hitoshi Miyaoka, MD
}

\begin{abstract}
Background There may be an increased risk of pulmonary thromboembolism (PTE) with antipsychotic drugs, so this association was investigated in autopsy cases of sudden unexpected death determined by the Department of Legal Medicine of a Japanese university hospital.

Methods and Results Records of 1,125 forensic autopsies (808 males, 317 females) performed during the study period for investigation of the cause of sudden unexpected death were reviewed and a logistic regression analysis was performed to explore whether age, gender, body mass index (BMI), and antipsychotic drug use were associated with fatal PTE. Among all records, 34 (3.0\%; 14 males, 20 females) indicated the use of antipsychotic drugs and 28 (2.5\%; 9 males, 19 females) indicated PTE as the cause of death. Of the 28 subjects who died from PTE, 8 had taken antipsychotic drugs (29\%) and all were female. Female gender and antipsychotic drug use accounted for a significantly higher risk of PTE death with an odds ratio of 4.22 (95\% confidence interval (CI), 1.82-9.78; $\mathrm{p}<0.01$ ) and 10.49 (95\% CI, 3.95-27.85; $\mathrm{p}<0.01$ ), respectively.

Conclusions Japanese women taking antipsychotic drugs may be at particular risk for PTE. (Circ J 2004; 68:
\end{abstract} $850-852)$

Key Words: Antiphospholipid antibodies; Atypical antipsychotic drugs; Phenothiazines; Pulmonary thromboembolism

$\mathbf{I}$ $\mathrm{n}$ massive pulmonary thromboembolism (PTE), rapidly progressive pulmonary hypertension leads to right heart failure and possible sudden death. In the USA, acute PTE is the third most common acute cardiovascular disease after cardiac ischemic syndromes and stroke, ${ }^{1,2}$ and although the incidence of PTE is lower in Japan, it is gradually increasing?

In Western countries, including the USA, PTE has been reported in patients treated with either conventional antipsychotic drugs or atypical antipsychotic drugs including clozapine. This association of PTE with antipsychotic drugs is being recognized more frequently in Japan, partly because more autopsies are being performed and partly because clinical diagnostic techniques have improved. In our previous report we suggested that Japanese patients treated with atypical antipsychotic drugs such as risperidone, as well as those treated with conventional agents such as phenothiazines, may be at risk of acute PTE even if otherwise healthy. In the present study we assessed this risk by considering autopsy cases in which the cause of sudden unexpected death was determined by the Department of Legal Medicine at Kitasato University, a Japanese

(Received January 26, 2004; revised manuscript received June 23, 2004; accepted July 2, 2004)

Departments of Psychiatry, *Emergency and Critical Care Medicine, and **Legal Medicine, Kitasato University, School of Medicine and Department of Occupational Mental Health, Kitasato University, Graduate School of Medical Sciences, Sagamihara, Japan

Mailing address: Satoko Hamanaka, MD, Department of Psychiatry, Kitasato University, School of Medicine, 1-15-1 Kitasato, Sagamihara 228-8555, Japan.E-mail: lesalondusatoko@aol.com university hospital.

\section{Methods}

Records of autopsies performed from January 1998 to December 2002 for investigation of the cause of sudden unexpected death were reviewed. Family consent had been obtained for the autopsy. Cases of massive thrombus that filled the lumen of the major pulmonary vessels and without other findings related to the cause of death, such as lesions in the coronary arteries or aorta, were identified.

Age, gender, body mass index (BMI), and antipsychotic drug use were reviewed in all autopsied individuals. Psychiatric profiles, risk factors for thrombotic disease (recent surgery, trauma, heart disease, cardiovascular disease, neoplastic disease, systemic illness such as collagen vascular diseases and obesity), and anticardiolipin antibodies were investigated, especially in those who had taken antipsychotic drugs. Psychiatric diagnosis was based on the Diagnostic and Statistical Manual of Mental Disorders, edition IV (DSM-IV) $)^{5}$ and the criteria of obesity was a BMI $>30$.

\section{Statistic Analysis}

A logistic regression analysis was performed using SPSS (version 11.5; Chicago, ILL, USA) to explore whether age, gender, BMI, and antipsychotic drug use were associated with fatal PTE. Odds ratios (OR) are presented with $95 \%$ confidence interval (CI) and a p-value less than 0.05 was considered significant. 
Table 1 Results of Logistic Regression Analysis $(n=1,125)$ of the Autopsy Cases of Pulmonary Thromboembolism

\begin{tabular}{lcr}
\hline \hline Factors & Odds ratio $(95 \%$ CI) & p value \\
\hline Age (years) & $1.02(0.99-1.04)$ & 0.23 \\
Sex (male 0, female 1) & $4.22(1.82-9.78)$ & $<0.01$ \\
BMI & $1.06(0.99-1.13)$ & 0.08 \\
ASD & $10.49(3.95-27.85)$ & $<0.01$ \\
\hline
\end{tabular}

ASD, antipsychotic drug; BMI, body mass index; 95\%CI, 95\% confidence interval.

\section{Results}

Of 1,125 forensic autopsies (808 males, 317 females) performed during the study period, 34 records $(3.0 \% ; 14$ males, 20 females) indicated the use of antipsychotic drugs (32 were diagnosed schizophrenia, 1 schizoaffective disorder, 1 mental retardation). Of the 34 records, $28(2.5 \% ; 9$ males, 19 females) indicated PTE as the cause of death and of these 28 subjects, 8 had taken antipsychotic drugs (29\%); all were female, aged between 32 and 65 years $(55 \pm 11)$.

According to the logistic regression analysis, female gender and antipsychotic drug use accounted for a significantly higher risk of PTE death with OR of 4.22 (95\% CI, 1.82-9.78; $\mathrm{p}<0.01)$ and 10.49 (95\% CI, 3.95-27.85; $\mathrm{p}<$ 0.01 ), respectively (Table 1 ).

We further reviewed the records of the 8 subjects who had taken antipsychotic drugs (Table 2). Seven had schizophrenia and 1 had schizoaffective disorder. Chlorpromazine or other phenothiazines had been prescribed in 6 cases, haloperidol and other butyrophenones in 5, and atypical antipsychotic drugs such as risperidone in 4 . Seven had taken more than 2 antipsychotic drugs. Only 1 subject had a risk factor for thrombotic disease (collagen vascular disease) (Table 3). Five demonstrated massive thrombus that filled the lumen of both pulmonary arteries, 3 demonstrated massive thrombus that filled the trunk of the pulmonary artery and pulmonary infarction was not seen in any of the 8 subjects. Five demonstrated thrombi above the knees, 3 did not. None had been confined to bed or had difficulty walking. Two of the patients died in the hospital after cardiopulmonary arrest, and 6 were already dead at the time of discovery. Anticardiolipin antibody concentration in serum, which was kept frozen at $-80^{\circ} \mathrm{C}$ until tested, was investigated and all samples were negative.
Table 3 Characteristics of the 8 Female Autopsy Cases of Pulmonary Thromboembolism Associated With Antipsychotic Drugs

\begin{tabular}{ccccc}
\hline $\begin{array}{l}\text { Case } \\
\text { no. }\end{array}$ & $\begin{array}{c}B M I \\
\left(\mathrm{~kg} / \mathrm{m}^{2}\right)\end{array}$ & Riskfactors & Site of PTE & DVT \\
\hline 1 & 16.6 & $R A$ & $T$ & + \\
2 & 26.8 & & $R+L$ & - \\
3 & 25.6 & & $T$ & - \\
4 & 28.2 & $R+L$ & + \\
5 & 26.9 & $R+L$ & + \\
6 & 27.7 & $R+L$ & + \\
7 & 24.1 & $R+L$ & - \\
8 & 20.9 & $T$ & + \\
\hline
\end{tabular}

BMI, body mass index; DVT, deep vein thrombosis above the knee; RA, rheumatoid arthritis; $T$, trunk; $R$, right; L, left.

\section{Discussion}

In the present study, $2.5 \%$ of all forensic autopsy cases investigated by the Department of Legal Medicine of the School of Medicine at Kitasato University died of PTE. According to a similar study of the Tokyo Medical Examiner's Office, among 24,431 forensic autopsy cases in the past 10 years, from 1992 to 2001 , the cause of death in $215(0.88 \%$, female/male ratio $118 / 97$ or 1.25$)$ was PTE and the annual number of cases increased gradually. In addition, the percentage of PTE cases in the total number of autopsies increased by up to $1 \%$ from 19993 . We had previously found a disproportionately high number of patients with PTE who had taken antipsychotic drugs among all patients with PTE treated at an emergency center $(44 \%)^{4}$ and in our present study, $29 \%$ of all cases of fatal PTE had an association with antipsychotic drugs. Another report has shown showed similar rates of occurrence to those in the present study 6 and in the Netherlands, Vandenbroucke et al reported that among 14,000 autopsy cases investigated at Leiden University, 27 (1.9\%) were found to have PTE and of these $10(37 \%)$ had taken antipsychotic drugs? Thus, the association of PTE with antipsychotic drugs among all cases of PTE may be relatively high in countries with a lower overall incidence of PTE, such as the Netherlands and Japan.

The findings of the present study suggest that patients taking antipsychotic drugs have a higher risk for PTE, but an association of thromboembolic complications with conventional agents, including chlorpromazine, was suggested

Table 2 Psychiatric Profile of Autopsy Cases of Pulmonary Thromboembolism Associated With Antipsychotic Drugs

\begin{tabular}{|c|c|c|c|c|c|c|c|}
\hline \multirow{2}{*}{$\begin{array}{l}\text { Case } \\
\text { no. }\end{array}$} & \multirow{2}{*}{$\begin{array}{c}\text { Age } \\
\text { (years) }\end{array}$} & \multirow{2}{*}{ Gender } & \multirow{2}{*}{ Diagnosis } & \multicolumn{4}{|c|}{$A P D$} \\
\hline & & & & Phenothiazines & Butyrophenones & $S D A$ & Others \\
\hline 1 & 55 & $F$ & $S$ & $\begin{array}{l}\text { CP } 50 \mathrm{mg} \\
\text { Perphenazine } 16 \mathrm{mg}\end{array}$ & & & \\
\hline 2 & 64 & $F$ & $S$ & & & Risperidone $3 \mathrm{mg}$ & \\
\hline 3 & 51 & $F$ & $S$ & $\begin{array}{l}\text { CP } 175 \mathrm{mg} \\
L P 50 \mathrm{mg}\end{array}$ & $H P D 18 \mathrm{mg}$ & Risperidone $6 \mathrm{mg}$ & \\
\hline 4 & 65 & $F$ & $S$ & $\begin{array}{l}C P 150 \mathrm{mg} \\
L P 75 \mathrm{mg}\end{array}$ & $H P D 8 m g$ & & \\
\hline 5 & 52 & $F$ & $S A$ & & $H P D 1.6 \mathrm{mg}$ & Risperidone $8 \mathrm{mg}$ & Zotepine $50 \mathrm{mg}$ \\
\hline 6 & 32 & $F$ & $S$ & $\begin{array}{l}\text { CP } 12.5 \mathrm{mg} \\
L P 35 \mathrm{mg}\end{array}$ & $B P D 23 m g$ & & Zotepine $150 \mathrm{mg}$ \\
\hline 7 & 54 & $F$ & $S$ & CP $37.5 \mathrm{mg}$ & & $\begin{array}{l}\text { Risperidone } 3 \mathrm{mg} \\
\text { Perospirone } 24 \mathrm{mg}\end{array}$ & \\
\hline 8 & 63 & $F$ & $S$ & CP $25 \mathrm{mg}$ & $H P D 4 m g$ & & \\
\hline
\end{tabular}

APD, antipsychotic drug; SDA, serotonine-dopamine antagonist; S, schizophrenia; SA, schizoaffective disorder; CP, chlorpromazine; $L P$, levomepromazine; HPD, haloperidol; BPD, bromperidol. 
more than 20 years ago ${ }^{8,9}$ Zornberg and Jick have also reported that low-potency antipsychotic drugs such as chlorpromazine and thioridazine were more strongly associated with venous thromboembolism than were highpotency antipsychotic drugs such as haloperidol ${ }^{10}$ and recently, thromboembolic complications have been reported with clozapine, an atypical antipsychotic drug?,11,12 In the present study, 7 of the 8 cases of PTE associated with antipsychotic drugs involved conventional antipsychotic drugs and 4 cases potentially involved atypical antipsychotic drugs such as risperidone or perospirone. Although half of the patients had taken atypical antipsychotic drugs, it was not possible to determine which type of antipsychotic drugs was more strongly associated with PTE.

Mechanisms involved in the thrombotic complications associated with antipsychotic drugs, such as the lupus-like syndromes induced by phenothiazines or atypical antipsychotic drugs, have been suggested ${ }^{13,14}$ However, Canoso and Oliviera followed psychiatric patients treated longterm with chlorpromazine in whom concentrations of antiphospholipid antibodies was elevated and could not find an increase in the incidence of thrombosis $!^{15}$ In our previous and present studies, anticardiolipin antibody could not be detected in the cases of PTE involving antipsychotic drugs. Atypical antipsychotic drugs have an affinity for the type$2 \mathrm{~A}$ serotonin receptor (5HT2A) that is at least 10 -fold greater than for the type-2 dopamine receptor (D2) ${ }^{16}$ and it has been suggested that 5HT2A-induced platelet aggregation might be affected in patients receiving risperidone or clozapine 4

The results of the present study also suggest that female patients have a higher risk for PTE because all the PTE subjects associated with antipsychotic drugs were female. Therefore, a hormonal effect should be considered. The most notorious hormonal adverse effect of antipsychotic drugs is hyperprolactinemia, which is mediated by the dopamine-blocking action of these agents. Wallaschofski et al suggest that hyperprolactinemia is an important novel risk factor for PTE in patients on antipsychotic drugs and that the thrombogenic effect is mediated through enhanced platelet reactivity 17 This adverse effect is also seen in male patients, but is more marked in female patients! ${ }^{18}$ Unfortunately, we did not examine the prolactin concentrations in the present study and we wish to further investigate the hormonal effects in cases of PTE associated with antipsychotic drugs.

In the present study, 7 of the 28 PTE subjects suffered from schizophrenia and therefore the risk for PTE of schizophrenia itself should be considered. Kaiya et al have reported that it is associated with increased platelet aggregation, ${ }^{19}$ but other reports suggest there is an increased risk for PTE for patients with schizophrenia who are taking conventional antipsychotic drugs, including chlorpromazine, than for those who are untreated9,20,21 Zornberg and Jick demonstrated that antipsychotic therapy, including the atypical drugs, may increase the risk for PTE by an OR of 7.1, but that schizophrenia alone did not ${ }^{10}$

\section{Conclusion}

In Japan, female patients taking antipsychotic drugs may have an increased risk for PTE.

\section{References}

1. Kumasaka N, Sakuma M, Shirato K. Incidence of pulmonary thromboembolism in Japan. Jpn Circ J 1999; 63: 825-827.

2. Nakamura M, Okada O, Sakuma M, Nakanishi N, Miyahara Y, Yamada N, et al. Incidence and clinical characteristics of chronic pulmonary thromboembolism in Japan compared with acute pulmonary thromboembolism: Results of a multicenter registry of the Japanese Society of Pulmonary Embolism Research. Circ J 2002; 66: $257-260$.

3. Tanifuji T, Kageyama N, Ro A, Hamamatsu A, Misawa S. A histopathological study of fatal pulmonary thromboembolism and deep vein thrombosis: In the study of deep vein thrombosis amoun forty patients with pulmonary thromboembolism Jpn J Phlebol 2003; 14: $189-195$.

4. Kamijo Y, Soma K, Nagai T, Kurihara K, Ohwada T. Acute massive pulmonary thromboembolism associated with risperidone and conventional phenothiazines. Circ J 2003; 67: 46-48.

5. American Psychiatric Association. Diagnostic and Statistical Manual of Mental Disorders, edition IV. American Psychiatric Association, Washington D.C., 1994.

6. Hagg S, Spigset O, Soderstrom TG. Association of venous thromboembolism and clozapine. Lancet 2000; 355: 1155-1156.

7. Vandenbroucke JP, Bertina RM, Holmes ZR, Spaargaren C, van Krieken JH, Menten B, et al. Factor V Leiden and fatal pulmonary embolism. Thromb Haemost 1998; 79: 511-516.

8. Ungvari G. Neuroleptic-related sudden death (proven or a mere hypothesis?). Pharmacopsychiatry 1980; 13: 29-33.

9. Haefner H, Brehm I. Thromboembolic complications in neuroleptic treatment. Compr Psychiatry 1965; 6: 25-34.

10. Zornberg GL, Jick H. Antipsychotic drug use and the risk of firsttime idiopathic venous thromboembolism: A case-control study. Lancet 2000; 356: 1219-1223.

11. Walker AM, Lanza LL, Arellano F, Rothman KJ. Mortality in current and former users of clozapine. Epidemiology 1997; 8: 671-677.

12. Lacika S, Cooper JP. Pulmonary embolus possibly associated with clozapine treatment. Can J Psychiatry 1999; 44: 396-397.

13. Thomassen R, Vandenbroucke JP, Rosendaal FR. Antipsychotic medication and venous thrombosis. Br J Psychiatry 2001; 179: 63-66.

14. Davis S, Kern HB, Asokan R. Antiphospholipid antibodies associated with clozapine treatment. Am J Hematol 1994; 46: 166-167.

15. Canoso RT, Oliviera RM. Chlorpromazine-induced anticardiolipin antibodies and lupus anticoagulant: Absence of thrombosis. Am J Hematol 1998; 27: 272-275.

16. Love RC. Novel versus conventional antipsychotic drugs. Pharmacotherapy 1996; 16: $6 \mathrm{~S}-10 \mathrm{~S}$.

17. Wallaschofski H, Eigenthaler M, Kiefer M, Donne M, Hentschel B, Gertz HJ, et al. Hyperprolactinemina in patients on antipsychotic drugs causes ADP-stimulated platelet activation that might explain the increased risk for venous thromboembolism: Pilot study. J Clin Psychopharmacol 2003; 23: 479-483.

18. Kinon BJ, Gilmore JA, Liu H, Halbreich UM. Prevalence of hyperprolactinemia in schizophrenic patients treated with conventional antipsychotic medications or risperidone. Psychoneuroendocrinology 2003; 28: 55-68

19. Kaiya H, Imai H, Muramatsu Y, Nozaki M, Fujimura H, Adachi S, et al. Platelet aggregation response in schizophrenia and prostaglandin E1. Psychiatry Res 1983; 9: 309-318.

20. Grahmann H, Suchenwirth R. Thrombosis hazard in chlorpromazine and reserpine therapy of endogenous psychosis. Nervenarzt 1959; 30: $224-225$.

21. Meier-Ewert K, Baumgart HH, Friedenberg P. Thromboembolism complications in neuro- and thymoleptic therapy. Dtsch Med Wochenschr 1967; 92: 2174-2178. 